\title{
Status and performance of the CMS muon system in Run2
}

\author{
Andres Cabrera-Mora* \\ Universidad de los Andes (CO) \\ E-mail: andres.leonardo.cabrera.mora@cern.ch
}

The CMS muon system has played a key role for many physics results obtained from the LHC Run1 and Run2 data. During the Long Shutdown (2013-2014), as well as during the last year-end technical stop (2015-2016), significant consolidation and upgrades have been carried out on the muon detectors and on the Level-1 (L1) muon trigger. The algorithms for muon reconstruction and identification have also been improved for both the High-Level Trigger (HLT) and the offline reconstruction. Results of the performance of muon detectors, reconstruction and trigger, obtained using data collected at $13 \mathrm{TeV}$ center-of-mass energy during the 2015 and 2016 LHC runs, are presented. Comparison of simulation with experimental data is also discussed where relevant. The system's state of the art is also shown, as well the improvements foreseen to achieve excellent overall quality of muon reconstruction in the high-luminosity conditions expected during Run2.

38th International Conference on High Energy Physics

3-10 August 2016

Chicago, USA

\footnotetext{
* Speaker.

${ }^{\dagger}$ on behalf of the CMS collaboration
} 


\section{The CMS Muon System Upgrade for Run2}

Detecting muons is one of the most important tasks of the CMS experiment [1], the presence of muons in the final state of a proton proton collision is almost always a sign of interesting physics. It is expected that muons are produced in the decay of a number of potential new particles, and in particular, one of the most cleanest signatures of the Higgs Boson is its decay to four muons.

The CMS muon system consists of gaseous detectors with three different technologies to identify muons and measure their momenta. The Drift Tubes (DT) are used for accurate measurement of the trajectory of muons in the central region of the barrel $(|\eta|<1.2)$. The Cathode Strip Chambers (CSC) are used for the same purpose in the endcap region $(0.9<|\eta|<2.4)$. The Resistive Plate Chambers (RPC) are installed in both the barrel and the endcap region $(|\eta|<1.6$ for L1 trigger and $|\eta|<1.8$ for reconstruction) to provide a fast signal when there is a muon passing through them. The fast signal generated by the RPC is used to activate the DTs, CSCs and the overall data acquisition system of the CMS experiment [2].

For muon triggering purposes, the CMS trigger is staged in two levels. The Level-1 Trigger (L1) [3], that is hardware-based and uses information of the muon system exclusively. And the High Level Trigger (HLT) [4], that is software based and combines information from muon system and inner tracker to identify and reconstruct muons. Information from the calorimeters is also used to compute muon isolation.

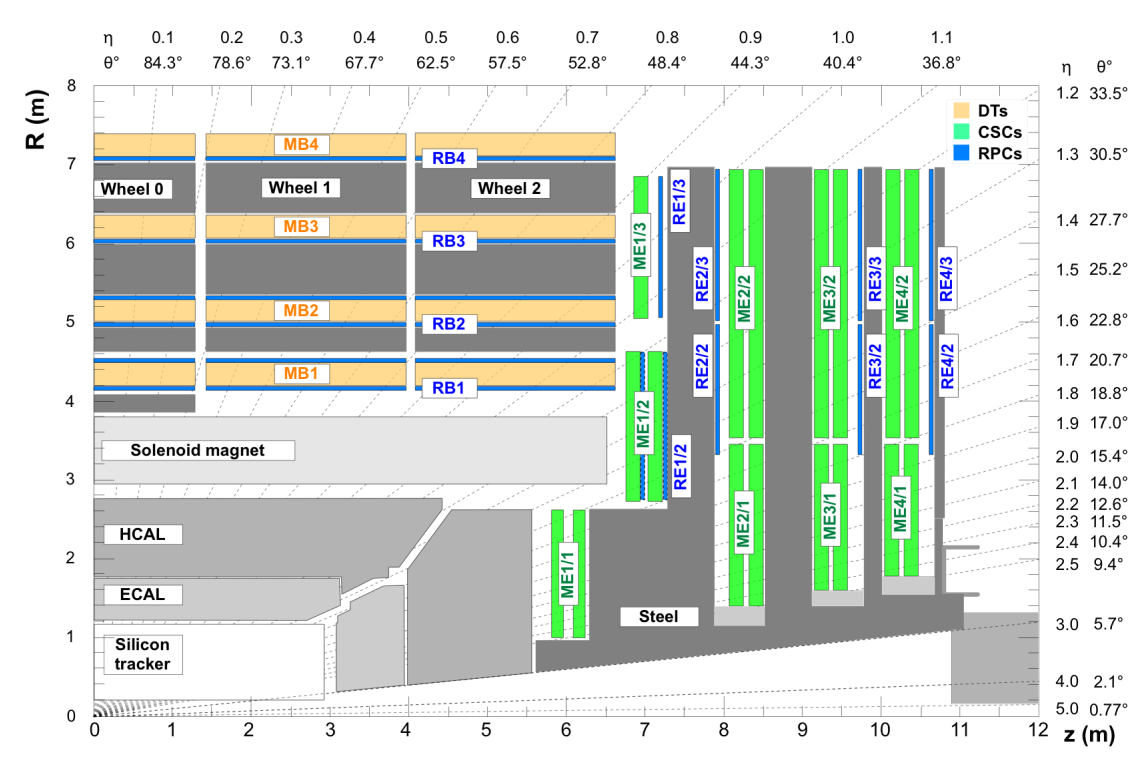

Figure 1: Cross section of a quadrant of the CMS detector [5].

During the long shutdown of the LHC in 2013 and 2014 several upgrades have been performed in the muon detectors. The CSC have added 72 additional chambers (36 in the negative endcap and 36 in the positive), which correspond to the ME4/2 station in Figure 1. Also the chamber electronics of the ME1/1 station has been updated improving the dectector granularity ( 48 channels to read 48 strips, before there were only 16 channels for the 48 strips). The RPC have added 144 additional chambers (72 in the negative endcap and 72 in the positive), corresponding to the RE4/2 and RE4/3 
stations in Figure 1. With those upgrades in the CSC and RPC the system redundancy is increased with 4 stations in the endcaps, this implies higher efficiency for triggers requiring 3 stations in the L1 muon trigger. Also this gives the possibility to tighten the trigger quality requirements that will imply a reduction on the rate, keeping a reasonably low threshold with a high instantaneous luminosity. The DT made a relocation of readout and trigger electronics in the service cavern (out of the experimental cavern) that opens the possibility to perform interventions during running time and also allows to develop improved trigger primitive algorithms.

\section{Muon reconstruction and Identification improvements}

A small reduction of muon reconstruction efficiency in the tracker, that increased with pileup, was observed in 2012 data. In order to recover it, two additional muon- specific tracking iterations have been implemented for Run2:

1. An Outside-in iteration, seeded from the muon system, designed to recover the missing muontrack in the tracker.

2. An Inside-Out iteration designed to re-reconstruct muon-tagged tracks with looser requirements to improve the hit-collection efficiency.

In addition to recovering the small inefficiency, the new algorithm has also improved the muon track quality, in particular the number of hits per track. This is reflected in the identification efficiency of standard selections as the Tight Muon selection [6].

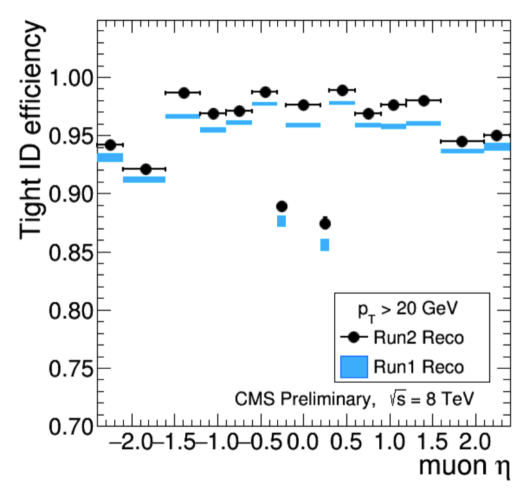

Figure 2: Efficiency as a function of muon pseu- Figure 3: Efficiency as a function of the number of recondorapidity for muon $P_{T}>20 \mathrm{GeV}$

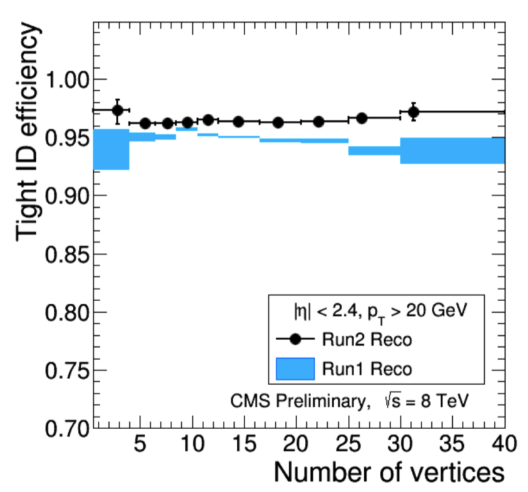

Tag-and-Probe efficiencies for Tight Muon ID on $\mathrm{Z} \rightarrow \mu \mu$ events from a subsample of data recorded in the last part of the 2012 Run, reconstructed with both the Run1 and Run 2 reconstruction algorithms are shown in Figures 2 and 3. The Run2 reconstruction improves the average efficiency by $1-2 \%$, in particular in events with high multiplicity of proton- proton interactions per bunch crossing (pileup) [6].

\section{Muon trigger improvements in HLT}

The muon triggers were updated with respect to the 2012 configuration. Single muon and Double muon trigger paths were improved, both in the online reconstruction and in the selection 
and quality cuts applied [5]. In particular, for the Single muon triggers, the tracking algorithms and pileup mitigation techniques were improved. Isolation computation was updated from single isolation cut (combining tracker and calorimeter information) to sequential isolation cuts, tuned independently for tracker and calorimeter components. For the Double muon triggers, a loose track based relative isolation cut was also added to both legs of the high $\mathrm{p}_{T}$ trigger, to reduce rates and keep thresholds identical to the ones used at the end of Run1.

\section{CMS Muon Detectors Performance}

Muon systems are efficiently taking high-quality data. The efficiency for the different subdetectors is calculated as the ratio between the number of detected and the number of expected hits. Figure 4 shows the efficiencies of the RPC chambers for both Barrel and Endcap, respectively. Figures 5 and 6 show the efficiencies for DT and CSC chambers respectively.
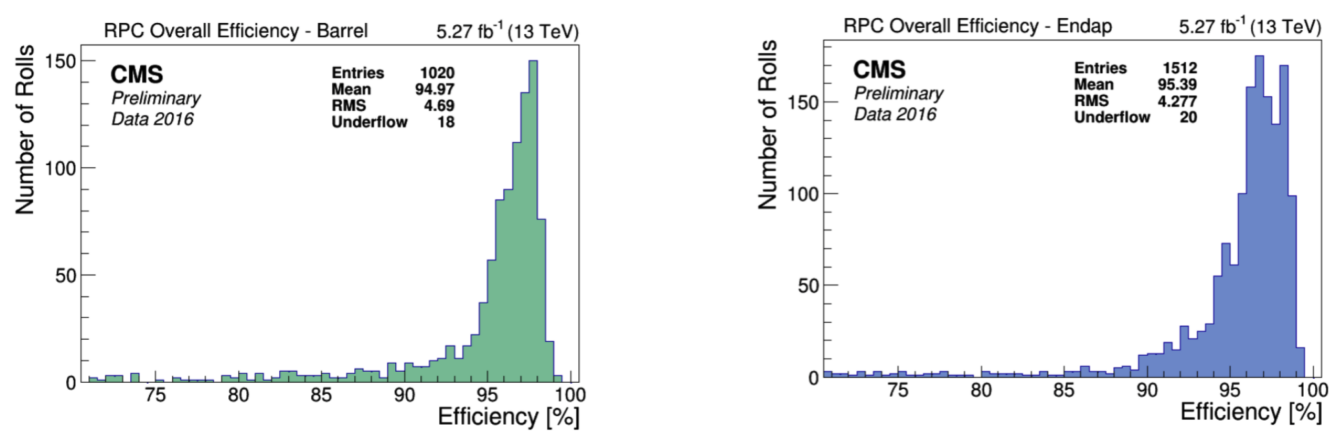

Figure 4: Efficiency (\%) of each RPC in the CMS Barrel (left) and Endcap (right) Muon detector to provide reconstructed hits [7].
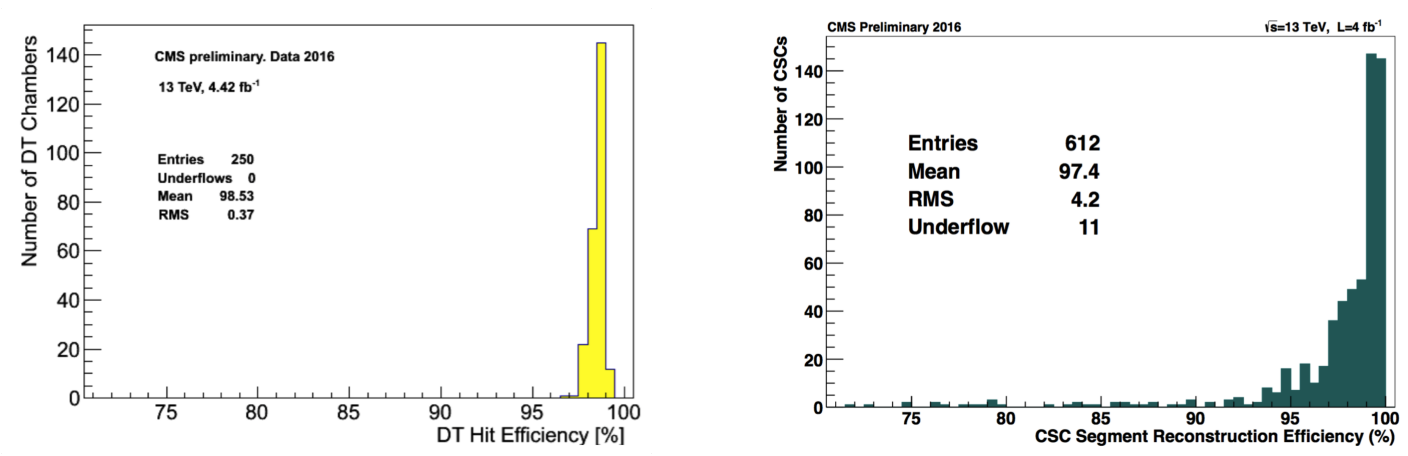

Figure 5: Efficiency (\%) of each DT chamber in the Figure 6: Efficiency (\%) of each CSC in the CMS CMS Barrel Muon detector to provide reconstructed Endcap Muon detector to provide reconstructed muon hits [7].

track segments [7].

\section{Dimuon invariant mass spectrum}

Figures 7 and 8 show the dimuon invariant mass spectrum in the vicitnity of the $\mathrm{J} / \psi$ meson and $\Upsilon(\mathrm{ns})$ meson respectively with 2016 data. These plots show the high accuracy for the reconstruction of the different dimuon invariant mass resonances using the muon system of the CMS experiment. 


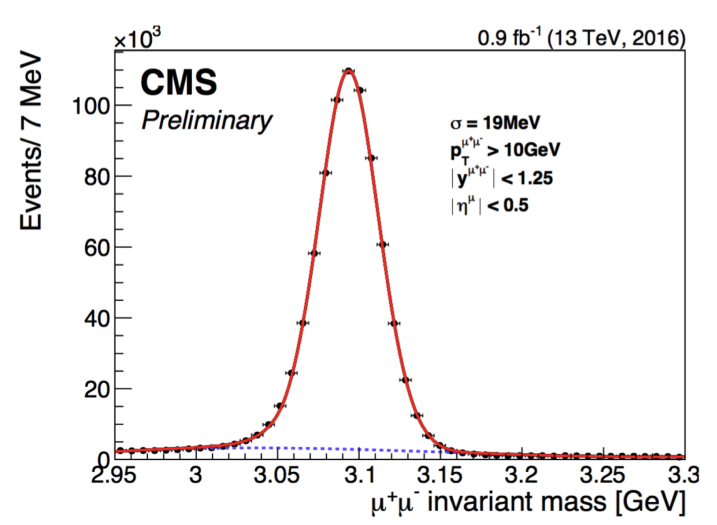

Figure 7: Dimuon invariant mass spectrum in the vicitnity of the $\mathrm{J} / \psi$ meson [8].

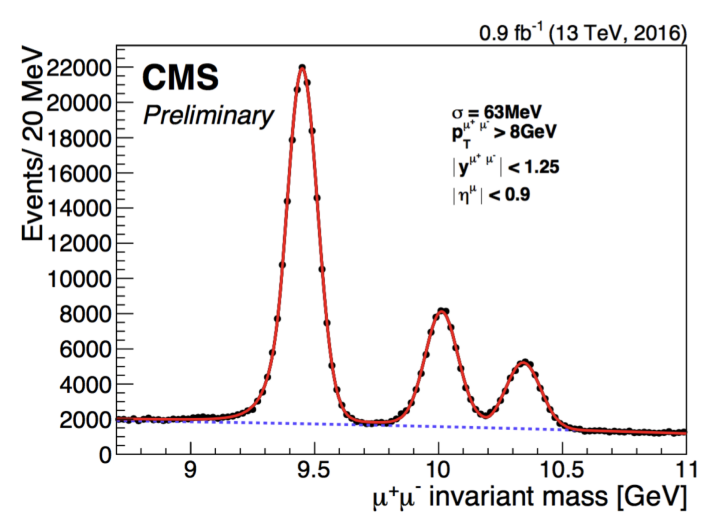

Figure 8: Dimuon invariant mass spectrum in the vicitnity of the $\Upsilon(\mathrm{ns})$ meson [8].

\section{Acknowledgements}

We thank the CERN accelerator department for its efforts to provide an excellent LHC performance and the CMS collaboration for the outstanding operation of the CMS detector. In addition, we thank the administrative department of science, technology and innovation of Colombia (COLCIENCIAS) for the financial support provided.

\section{References}

[1] CMS Collaboration, The CMS experiment at the CERN LHC, JINST 3 (2008) S08004.

[2] CMS Collaboration, The performance of the CMS muon detector in proton-proton collisions at $\sqrt{s}=$ 7 TeV at the LHC, JINST 8 (2013) P11002.

[3] CMS collaboration, The TriDAS project, technical design report. Volume 1: The level-1 trigger, CERN-LHCC-2000-038, http://cdsweb.cern.ch/record/706847.

[4] CMS collaboration, The TriDAS project, technical design report. Volume 2: Data acquisition and high-level trigger technical design report, CERN-LHCC-2002-026, http://cdsweb.cern.ch/record/578006.

[5] G. Abbiendi for the CMS Collaboration, The CMS muon system in Run2: preparation, status and first results. Proceedings, 2015 European Physical Society Conference on High Energy Physics (EPS-HEP 2015). [arXiv:1510.05424].

[6] CMS Collaboration, Muon Reconstruction and Identification Improvements for Run-2 and First Results with 2015 Run Data, CMS DP-2015/015.

[7] CMS Collaboration, Performance of the CMS Muon Detectors in 2016 collision runs, CMS DP-2016/046.

[8] CMS Collaboration, Muon performance in early 2016 collision data, CMS DP-2016/027. 